DOE/ER/ $45238--8$

DE92 009989

\title{
PROPERTIES OF MOLECULAR SOLIDS AND FLUIDS AT HIGH PRESSURE AND TEMPERATURES
}

\section{Progress Report}

for period July 1, 1989 - July 1, 1992

Richard D. Etters

Physics Department

Colorado State University

Fort Collins, Colorado $\mathbf{8 0 5 2 3}$

March 1992

Prepared for

The U.S. Department of Energy

Agreement No. DE-FG02-86ER45238

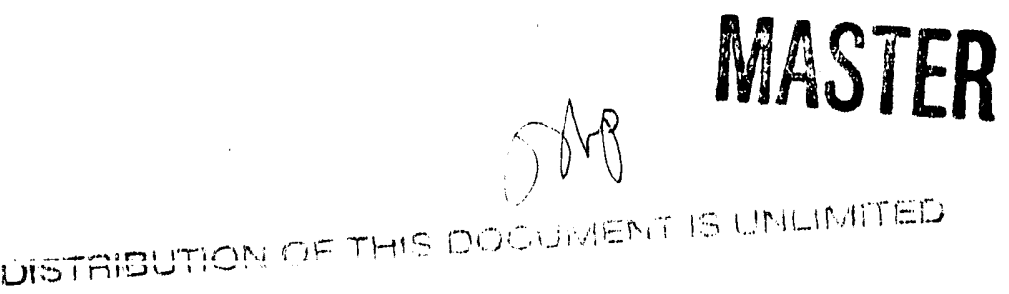




\begin{abstract}
This renewal request ${ }^{*}$ for DOE grant DE-FG02-86ER45238, is dedicated to providing a complete thermodynamic profile of solids, fluids, and fluid mixtures, over a wide range of temperatures and pressures. We are partially motivated by technological interest in detonation, combustion, superhard high pressure materials, and high temperature superconductors, which are important components of interest at various DOE laboratories.

Our work on fluids and solids, composed of simple molecules, involves the determination of structures, phase transitions, pressure-volume relations, phonon, vibron, ana libron modes of excitation, sound velocities, specific heats, thermal expansion, virial coefficients, sublimation energies, and orientational, translational, and magnetic correlations.
\end{abstract}

We hope that the study of these systems under extreme thermodynamic conditions will lead to exotic new materials of value, as well as enhanced fundamental understanding.

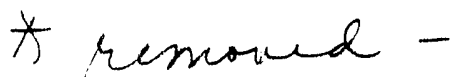

\title{
DISCLAIMER
}

This report was prepared as an account of work sponsored by an agency of the United States Government. Neither the United States Government wor any agency thereof, nor any of their employees, makes any warranty, express or implied, or assumes any legal liability or responsibility for the accuracy, completeness, or usefulness of any information, apparatus, product, or proess disclosed, or represents that its use would not infringe privately owned rights. Reference herein to any specific commercial product, process, or service by trade name, trademark, manufacturer, or otherwise does not necessarily constitute or imply its endorsement, recommendation, or favoring by the United States Government or any agency thereoi. The views United States Governors expressed herein do not necessarily state or reflect those of the 


\section{PROGRESS REPCRT}

In this section we first list articles and books published, submitted, or nearly finished during the grant period $7 / 1 / 89$ to present. They will then be discussed in groups with most attention to those not yet in the open literature.

1. B. Kuchta and R. D. Etters, "Calculated properties of solid $\mathrm{N}_{2} \mathrm{O}$ at various temperatures and pressures," J. Chem Phys. 95 , $5 \overline{399}$ (1991).

2. B. Kuchta and R. D. Etters, "A method for calculating solid-solid phase transitions at high temperatures: Application to $\mathrm{N}_{2} \mathrm{O}$," Phys. Rev. B 45, 1 March (1992).

3. B. Kuchta, R. D. Etters, and R. Lesar, "The influence of molecular shapes on the relative stability of solid phases: application to $\mathrm{N}_{2} \mathrm{O}, " \mathrm{~J}$. Chem. Phys., submitted (1992).

4. R. D. Etters and B. Kuchta, "static and dynamic properties of solid $\mathrm{CO}_{2}$ at various temperatures and pressures," J. Chem. Phys. 90, 45.37 (1989). 
5. B. Kuchta and R. D. Etters, "Calculated high pressure properties of solids composed of non-centrosymmetric mr lscules, Erontiers of high pressure Essearch, D. Hochheimer and R. D. Etters, eds., Plenum Press, New York, Vol. 286, March (1992).

6. B. Kuchta and R. D. Etters, "Free energy calculation of the cubic to orthorhombic.transition of $\mathrm{CO}_{2}$ at room temperature," J. Chem. Phys., submitted (1992).

7. B. Kuchta and R. D. Etters, "Features of the Histogram Monte Carlo Method: Application to $\mathrm{N}_{2}$ melting on graphite," J. Comp. Phys., submitted 24 Feb. (1992).

8. J. Belak, R. Lesar, and R. D. Etters, "Calculated thermodynamic properties and phase transitions of solid $\mathrm{N}_{2}$ at temperatures $0 \leq T \leq 300 \mathrm{~K}$ and pressures $0 \leq \mathrm{P} \leq 100 \mathrm{GPa}$," $\mathrm{J}$. Chem. Phys. 92, 5430 (1990).

9. B. Kuchta and R. D. Etters, "On the $\alpha-\gamma-\varepsilon$ transition in solid $\mathrm{N}_{2}, "$ in progress $(1992)$.

10. B. Kuchta and R. D. Etters, "Thermodynamic properties of solid

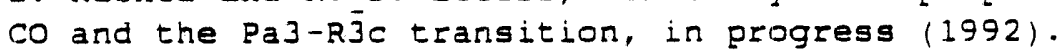

11. B. Kuchta, T. Luty, and R. D. Etters, "On the nature of the orientational transition in crystalline A-TCNB," J. Chem. Phys. 93, 5935 (1990).

12. R. D. Etters, "Matrix isolation study nf atomic hydrogen impurities in solid $\mathrm{H}_{2}$ and the rare gases," in progress $(1992)$.

13. Frontiers of high pressure research, edited by $D$. Hochheimer and R. D. Etters, Vol. 286, Plenum Press, New York, March $(1992)$.

14. R. D. Etters, "Structures and phase transitions of simple molecular solids," in simple molecular solids at very high density, edited by A. Polian, P. Loubeyre, and N. Boccara, Plenum Press, N.Y., P. 109 (1989).

A. $\mathbf{N}_{2} \mathrm{O}$

In (1) a multi-dimensional energy optimization method was used to determine the equilibrium structures in the limit of zero temperature. The calculated enthalpies showed that the pa3 structure is stable for pressures $P \leq 4.75$ GPa, above which the orthorhombic Cmca phase prevailed. The volume change on transition was $\Delta V=0.25 \mathrm{~cm}^{3} / \mathrm{mole}$. In 
collaboration with this work, $x$-ray measurements ${ }^{l y}$ at LANL found the orthorhombic transition at $P=4.85 \mathrm{GPa}$, with $\Delta V=0.27 \mathrm{~cm}^{3} / \mathrm{mole}$. Constant pressure Monte Carlo, with periodic, deformable boundary conditions, was used to determine the lattice parameters, internal energies, and $\mathrm{P}-\mathrm{V}$ relations in both phases, at $\mathrm{T}=0$ and $300 \mathrm{~K}$. Lattice dynamics was used to calculate the libron frequencies at various pressures in both phases, and second virial coefficients were determined. All calculated properties are in good agreement with experiment. $18,21-23$

The $x$-ray diffraction measurements ${ }^{18}$ at 100 and $300 \mathrm{k}$ suggest that the cubic to orthorhombic transition pressure is independent of temperature at $P_{1}=4.85 \mathrm{GPa}$, and our zero temperature result supports that view. In (2) we decided to test this argument by calculating $P_{\text {, at }}$ room temperature. Unfortunately, the Monte Carlo method was not useful here because the two observed phases and a competing tetragonal structure are metastable over the entire pressure range of interest. This was determined to be because of a large orientational potential barrier along the reaction path. Thus, the only option was to calculate the relative Gibbs free energies between competing phases, which is simple at low $T$ where the entropy term can be neglected, but not necessarily at high $T$. To solve this problem we developed a generalization of a promising technique $24-26$ not previously applied to a solid-solid transition in a physical system. A reversible thermodynamic path is constructed by considering a Hamiltonian for which the internal energy is

$$
U_{i}(\lambda)=\lambda U_{i}+(1-\lambda) U_{m}
$$


where $U_{i}$ is the internal energy of the $i^{\text {th }}$ phase, and $U_{m}$ is that for an exactly solvable model system. As the control parameter $\lambda$ varies from zero to unity, the system continuously transforms from the model to the physical system $i$. The following exact thermodynamic relations apply.

$$
\begin{aligned}
& \partial F_{i}(\lambda) / d \lambda=\left\langle\partial U_{i}(\lambda) / d \lambda\right\rangle=\left\langle U_{i}-U_{m}\right\rangle \lambda \\
& F_{i}(V)=F_{m}(J)+\int_{0}^{1}\left\langle\partial U_{i}(\lambda) / d \lambda\right\rangle d \lambda \\
& F_{i}(V)=F_{i}\left(V_{0}\right)-\int_{0}^{V} P_{i}(V) d V \\
& G(P)=F+P V
\end{aligned}
$$

where $F, V, P$, and $G$ are the Helmholtz free energy, volume, pressure, and Gibbs free energy, respectively. The brackets $4-->$ indicate a thermal average, evaluated using a constant pressure Monte Carlo scheme. Utilizing these relationships, the phase transition occurs when the difference in Gibbs free energy between the cubic Fa. and orthorhombic Cmca structures is zero. That is,

$$
\Delta G(P)=\Delta F(P)+P \Delta V=0
$$

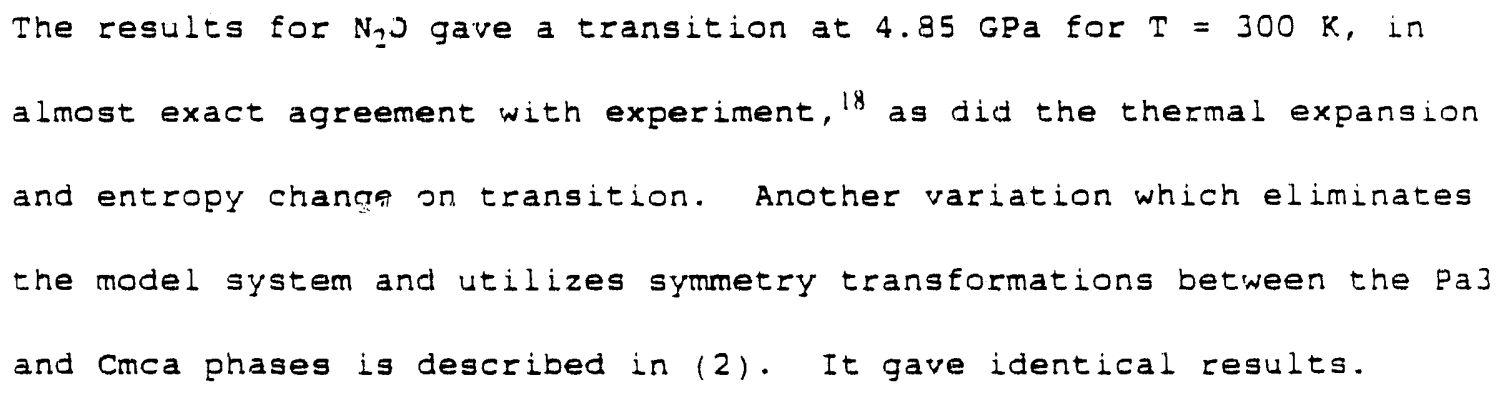


In (3) we describe the considerations required to develop an accurate representation of the interactions between $\mathrm{N}_{2} \mathrm{O}$ molecules. This work was done with Dr. R. Lesar of LANL. Calculation of the $\mathrm{N}_{2} \mathrm{O}$ charge distribution was decisive. Not only did it provide accurate values of the first four electric multipole moments, that strongly contribute to the $\mathrm{N}_{2} \mathrm{O}-\mathrm{N}_{2} \mathrm{O}$ interaction, but it also showed a substantial depression in the electronic charge distribution near the molecular midpoint. It was essential that this feature be reflected in the repulsive overlap terms of the interaction, which was accomplished using both isotropic and anisotropic ${ }^{19}$ site-site representations of the potential. For the latter case, this is the first application to a physical system. It was also shown that the same arguments used for $\mathrm{N}_{2} \mathrm{O}$ apply to $\mathrm{CO}_{2}$, as will be discussed in part $B$. Because $\mathrm{N}_{2} \mathrm{O}$ molecules are not centrosymmetric, experiment ${ }^{27,28}$ has suggested that they exhibit random end-to-end disorder in the pal phase at all solid temperatures. We tested this interpretation by introducing a random variable into our Monte Carlo calculation that randomized the invergion of the molecules. The average structure was Pa3, not only at $10 \mathrm{k}$ but also near melting. There was no reorientation from the initial end-to-end disorder at $10 \mathrm{k}$, and only a small amount near melting. The results are in agreement with experiment. 27.28

\section{B. Solid $\mathrm{CO}_{2}$}

In (4) we used a constant pressure Monte Carlo method, lattice dynamics, and classical perturbation theory to calculate the thernal expansion, pressure-volume relation at $300 \mathrm{~K}$, the temperature dependence of the zone center libron frequencies, and the pressure dependence of 
the three intramolecular vibron frequencies. These were found to be in good agreement with experiment. $29-33$

Evidence of a phase transition in solid $\mathrm{CO}_{2}$ comes from Raman measurements ${ }^{32,34}$ of the libron frequencies. At $80 \mathrm{k}$ they show a distinct change at $11 \mathrm{GPa}$ upon loading, and they change back to those appropriate for the Pa3 structure upon unloading between 2.5 and $4.5 \mathrm{GPa}$. This enormous hysteresis makes it impossible to extract an accurate value of the thermodynamic transition pressure. At $300 \mathrm{~K}$, Raman experiments ${ }^{32,34}$ show a trangition upon loading at 18 GPa, but no measurements were made upon unloading. Because of this uncertainty and because these measurements are not sufficient to identify the new phase, we asked Bart olinger of LANL to do $x$-ray diffraction measurements at $100 \mathrm{k}$. No transition was observed up to $11 \mathrm{GPa}$.

In the face of these confusing experimental results, we embarked upon a calculation to determine the pressure of this new transition and its structure. The Monte Carlo method was ineffective here for precisely the same reasons it is for $\mathrm{N}_{2} \mathrm{O}$, as described in II-A. This includes the same three competing metastable structures and the potential barrier. Consequently, calculation of the relative enthalpies revealed a trangition into the Cmca phase at $4.3 \mathrm{GPa}$, in the limit of zero temperature. In (6) we used a new variation of the previously described free energy method to calculate the transition pressure at $300 \mathrm{~K}$. A Cmca phase was predicted at $P_{l}=4.3 \pm ? .1 \mathrm{GPa}$. Thus, $d P_{1} / \mathrm{dT}$ appears constant over the range $0 \leq T \leq 300 \mathrm{~K}$, just as was establishedly for $\mathrm{N}_{2} \mathrm{O}$. We are not surprised at this or that $\mathrm{P}_{1}$ for the two solids are close, because $\mathrm{CO}_{2}$ and $\mathrm{N}_{2} \mathrm{O}$ are isoelectronic with the same mass, and have very similar charge distributions and $P-V$ relations. Moreover, both solids transform from $\mathrm{Pa} 3$ to the Cmca structure under pressure. 
Clearly, more experimental studies of this peculiar solid are warranted. We hope to persuade David Schiferl of LANL to do diamond cell studies on $\mathrm{CO}_{2}$.

\section{The Bistogran Method}

Part of our business is to develop new techniques that will prove useful in understanding the properties of molecular solids and fluids. An example of this is the histogram Monte Carlo Method described in (7), where it has recently been shown ${ }^{35}$ that information from a single thermodynamic point of a Monte Carlo simulation can provide valuable information about neighboring points. To see how this is so recall that for a canonical distribution the probability distribusion is

$$
P_{\beta}(E)=W(E) \exp [-\beta E+E]
$$

where the temperature is $\beta^{-1}=k T, W(E)$ is the density of states at energy $E$, and $F=f / \beta$ is the Helmholtz free energy. Note that $P_{j}(E)$ peaks at some energy $E_{o}(\beta)$ and tails off for energies away from this value. It is these tails that give information at energies relevant to neighboring points $\beta^{\prime}$. This is demonstrated by the exact expression,

$$
P_{\beta} \cdot(E)=\frac{P_{\beta}(E) \exp \left[-\left(\beta-\beta^{\prime}\right) E\right]}{\sum_{E} P_{\beta}(E) \exp \left[-\left(\beta-\beta^{\prime}\right) E\right]}
$$

An examination of this equation shows that $P_{B^{\prime}}(E)$ is accurate if $|\beta-\beta \cdot|$ is small and $P_{j}(E)$ is broad and well described. If this range is not sufficient to span the region of importance a straight forward generalization, called the multiple histogram method, ${ }^{35}$ can be used. Here probability distributions are calculated at $R$ different 
temperatures and these overlapping quantities extend the thermodynamic range over which accurate information can be obtained.

This latter method was used in (7) to calculate the melting

temperature $T_{M}$ of $N_{2}$ adlayers deposited on graphite, versus surface density $p$. Experiment shows that $T_{M}=47 \mathrm{~K}$ for all densities

$0.2 \leq p \leq 0.9$, where $\rho=1$ represents monolayer coverage. For $0.9<p \leq 1, T_{M}$ rapidly increases to about $85 \mathrm{~K}$. The calculations were remarkably successful and gave substantial insight into the melting process. This work could not have been done without the multiple histogram method, which took 25000 cpu hours on an IBM 6000 RISC work station.

A very efficient application of the histogram method was applied to the calculation of $\mathrm{P}_{\mathrm{t}}(300 \mathrm{~K})$ for $\mathrm{CO}_{2}$, described in II-B. It was shown in (6) that by calculating the probability distribution for the difference in enthalpy between the $\mathrm{Pa} 3$ and cmca states for different values of $\lambda$, the Gibbs free energy difference can be calculated directly, without the integration of Eq. (3).

\footnotetext{
D. Solid $\mathbf{N}_{2}$

The work reported in (8) is a tour du force in that properties of virtually every phase of $\mathrm{N}_{2}$, and the transitions between them, were calculated from a single ab initio representation of the inter-and intramolecular potentials. ${ }^{26}$ Constant pressure Monte Carlo with periodic, deformable boundary conditions, and classical perturbation theory, were used to determine vibron frequencies, P-V relations, thermal expansion coefficients, structures, phase transition pressuzes and temperatures, and various orientational order parameters. Particular attention was given the two plastic crystal phases that are
} 
often precursors to melting. For example, the orientationally

disordered, hexagonal $\beta$-phase was found to exhibit different

orientational behavior than previously suggested, $37-40$ but still

consistent with experiment. ${ }^{37,38}$ This work was done in collaboration

with J. Belak of LLNL and R. Lesar of LANL. Please refer to the

literature for further details.

In (9) we have been attempting to understand the low temperature

trangitions in solid $\mathrm{N}_{2}$, from the cubic Pa3 phase to the tetragonal

$P 4_{2} / \mathrm{mnm}$, structure at $P=0.32 \mathrm{GPa}$, and from the latter to the

rhombohedral $R \overline{3} \mathrm{c}$ phase at $20 \mathrm{GPa}$. Despite considerable effort the

stability of the tetragonal phase has never been satisfactorily

predicted. As discussed in (3), we believe the stability of various

phases is sensitive to the shape of the molecule, as represented by the

charge distribution. This feature must be reflected in the

intermolecular potential. While the isotropic site-site representation

of the potential, given by central forces between force centers on

different molecules, was very successful, it failed to predict the

$\mathrm{PL}_{2} / \mathrm{mnm}$ phase in $\mathrm{N}_{2}$. We have explored a subtle generalization of this

potential, using the anisotropic site-site representation of Price, 19

Here the forces between interaction sites on different molecules depends

on their relative orientation. This makes for a better representation

of the charge distribution and, for the first time, the stability of the

$P 4_{2} / \mathrm{mnm}$ phase is established at $0.32 \mathrm{GPa}$, as is observed. However, the

region of stability of the $R \overline{3} c$ structure, observed ${ }^{+1}$ above 20 GPa, is

now altered for the worse. It is intriguing that very small changes in

the anisotropy parameters can have a major influence on the relative

stability of various structures. We believe that a correct description 
of these three structures in $\mathrm{N}_{2}$ is a matter of fine tuning. Still, this remains to be established.

E. Solid co

We have been trying to understand solid co for a long time, partially because it is isoelectronic and has the same mass as $\mathrm{N}_{2}$. The phase diagrams of $\mathrm{N}_{2}$ and $\mathrm{CO}$ are strikingly similar but with oile important difference. Unlike $\mathrm{N}_{2}$, CO exhibits no tetragonal $\mathrm{P} 4_{2} / \mathrm{mnm}$ phase, and the transition to the $R \overline{3} c$ phase ${ }^{42}$ does not occur until $P$. $3.7 \mathrm{GPa}$. We have represented the co-co pair potential with the isotropic site-site expression ${ }^{36}$ used for $N_{2}$, with the multipoles replaced by those appropriate for $c o$. The results were very satisfactory, but the $\mathrm{Pa} 3$ to $R \overline{3} c$ transition could not be successfully established. The anisotropic site-site expression of Price ${ }^{19}$ discussed in IID, has been employed in (10) and the above mentioned transition is predicted very close to the observed pressure. Unfortunately, the P-V relations are not so good at high pressures. We believe that this can be rectified but, as usual, it must be proven.

\section{F. A-TCNB}

This organic solid is very interesting because an orientational order-disorder transition at $T_{O D}=210 \mathrm{~K}$ is complex and not easily characterized by simple models. At high temperatures A-TCNB forms ${ }^{+3, t 4}$ the space group $c_{2} / m$, with one complex per primitive unit cell. These molecules stack along the $\vec{C}$ axis, one upon the other, in alternate ab planes in a collinear orientation. Because of the interactions, the TCNB molecules are strongly localized in a nearly rigid orientational matrix. As the temperature is lowered to $T_{O D}$, the system transforms 
into a P2/a structure. The TCNB complexes arm undisturbed by the trangition and remain oriented in the ac plane. Experimental EPR, ${ }^{45}$ DF-ODMR, ${ }^{46}$ Raman, ${ }^{47} x$-ray, ${ }^{48}$ and neutron diffraction ${ }^{49}$ studies, have provided a mixed interpretation of the transition, as has been the case for theoretical calculations: $50-56$

Becauge of the quiescent response of the TCNB molecules, in (11) we determined that they form a static double well orientational ,yotential44;55 at euch A molecule site. This, modulated by the interactions between A molecules, characterize the transition. Using a constant pressure Monte Carlo and a susceptibility approach, we found the transition at $T=148 \mathrm{~K}$, some $60 \mathrm{~K}$ below experiment, but gatisfactory considering the uncertainty in the interactions. It was shown that the transition is convoluted in that sublattice exchange occurs in the herringbone lattice somewhat below Thl and orientational correlationg between planeg are also involved. Thro' the trangition is spread over a range of temperature around $T_{O}$ and shows both displacive and order-disorder features. The calculation seems to explain, at least qualitatively, all the experimental results.

\section{G. Atomic Hydrogen Impurities}

This work was partially motivated by renewed interest in develsping Eusion targets of molecular hydrogen enriched with atomic hydrogen impurities, ${ }^{57,58}$ which can enhance cross-sections by as much as $50 \%$. Among the recent experimental efforts in this direction, is the work of Clark sowers 59 and others at LLNL, and Jim Gaines ${ }^{60}$ at. the University of Hawaii. 
In (12) we have investigated the properties of dillute atomic hydrogen impurities in $\mathrm{H}_{2}$ and rare gas solid host lattices at pressure $0 \leq \mathrm{P} \leq 14 \mathrm{GPa}$. The wave function is given by

$$
\Phi=\varphi\left(\vec{r}_{0}-R_{0}\right) \prod_{i=1}^{N-1} \varphi\left(\vec{r}_{i}-\vec{R}_{i}\right) \prod_{j=1}^{N-1} f\left(\vec{r}_{0}-\vec{r}_{j}\right) \prod_{i<j}^{N i-1} f\left(r_{i j}\right)
$$

and the Hamiltonian is,

$$
H=-\frac{\hbar^{2}}{2 m} \nabla_{0}^{2}-\frac{\hbar^{2}}{2 m} \sum_{i=1}^{N-1} \nabla_{i}^{2}+\sum_{j=1}^{N-1} \nabla_{0}\left(r_{0 j}\right)+\sum_{i<j=1}^{N-1} U_{1}\left(r_{i j}\right)
$$

where the subscript zero refers to the impurity and $i=1, \ldots . N-1$ refers to the host lattice molecules. The vectorg $\left\{\vec{r}_{j}\right\}$ locate the positions of the molecules, and the equilibrium lattice positions are given by $\left\{\bar{R}_{i}\right\}$. The $\{\varphi\}$ act to spatially localize the molecules about their equilibrium positions and the $\left\{f\left(r_{i j}\right)\right\}$ account for important short ranged correlations between molecules. The potential between host lattice atoms is $U_{1}$ and $U_{0}$ is between an $H$ and a host lattice atom. Without going into mathematical detail, we have used a variational approach ${ }^{61}$ to minimize the expectation value of $\mathrm{H}$. Calculated are single particle distribution functions, the rms atomic fluctuations about equilibrium, the energy of the $H$ impurities in substitutional sites of the host lattice, and distortions of the lattice around the impurity. Results indicate that dilute $\mathrm{H}$ impurities should trap in all host lattices studied, and estimates of the temperature at which they will diffuse and recombine is in fair agreement with experimental evidence. $62-64$ Hyperfine and g-factor shifts are related to lattice distortions via the crystal field experienced by the H impurities, and experimental results ${ }^{62,65,66}$ are in reasonable agreement with our calculations. The 
calculations also show that substitutional absorption of $E$ is endoergic at low pressures and becomes excergic above 4 GPa, for all host lattices investigated.

\section{B. Overview Studies}

In (13): I was co-eritor of a book on recent advances in high pressure research and my postdoctorate presented an art.cle that gives an overview of problems involving solids compused of non-centrosymmetric molecules. In (14) we presented an overview of knowledge about solids composed of simple molecules at high presgure. An attempt was made to analyze these results in terms of a general. law of corresponding states. 20 

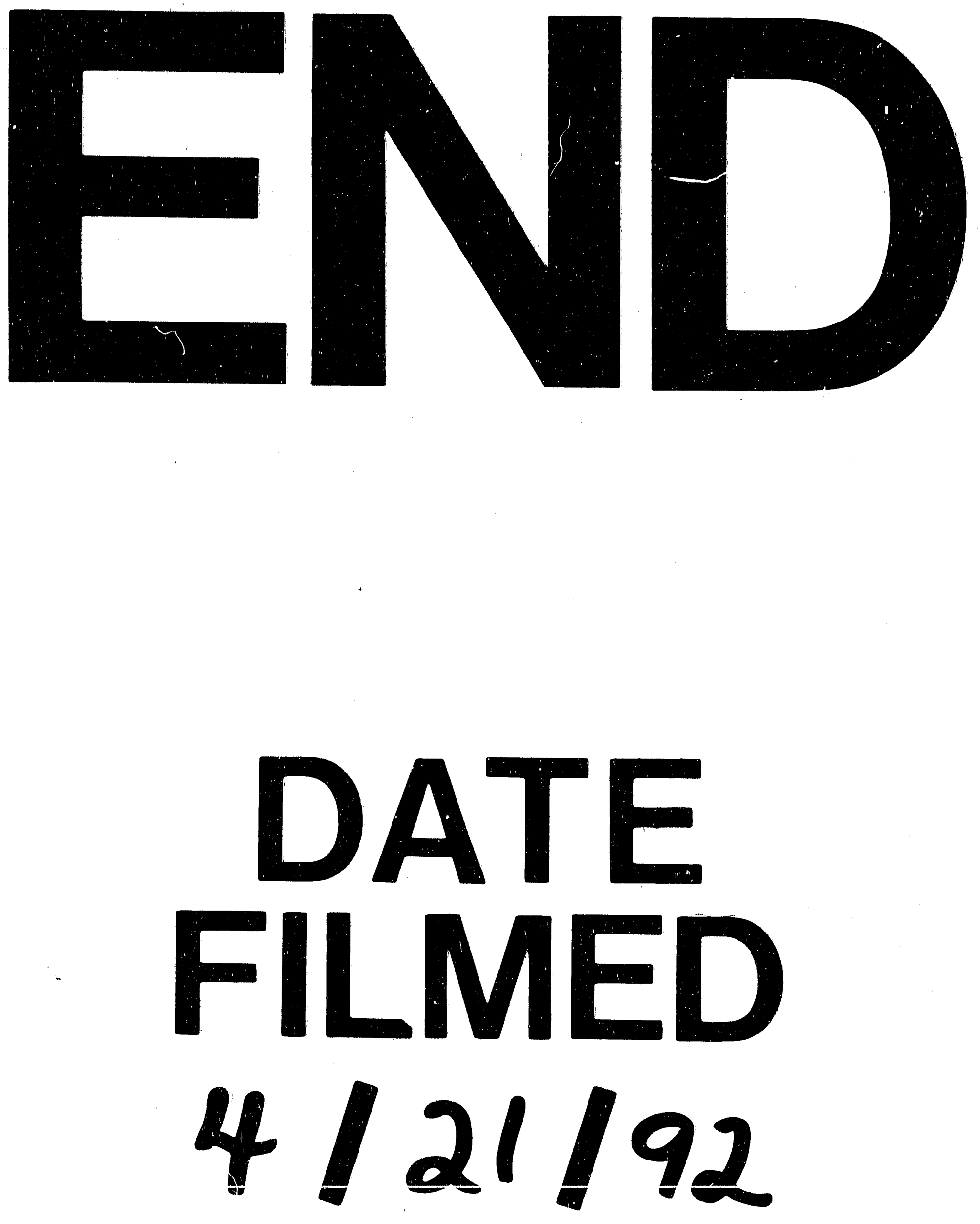
